\title{
Liquid Membrane Formulation for Removal of Kraft Lignin from Simulated Liquid Waste Solution
}

\author{
Norasikin Othman*, Ooi Zing Yi and Norlisa Harruddin \\ Centre of Lipid Engineering and Applied Research (CLEAR), Faculty of Chemical Engineering, \\ Universiti Teknologi Malaysia, 81310 UTM Skudai, Johor Bahru, Malaysia. \\ Received 11 December 2012, Revised 12 February 2013, Accepted 15 February 2013, Available online 25 February 2013
}

\begin{abstract}
Extraction of lignin from pulping wastewater as an external biofuel and in specialty chemicals will additionally benefit the chemical and automobile industries. Though lignin is nontoxic, it imparts brownish black colouration to the water bodies can cause environmental problems. Emulsion liquid membrane (ELM) technology is proposed to extract and recover the kraft lignin (KL) compound from the aqueous waste solution. This study investigated on development of a liquid membrane formulation for lignin removal from aqueous waste solution. Several parameters such as types and concentration of carrier, types of diluents and types of stripping agentswere studied. The results showed that the most favorable liquid membrane formulation is using Aliquat 336 as extractant, kerosene as diluents and sodium bicarbonate $\left(\mathrm{NaHCO}_{3}\right)$ as stripping agent which is almost $93 \%$ of lignin was extracted.
\end{abstract}

| Kraft lignin | Formulation | Liquid-liquid Extraction | Liquid Membrane | Wastewater |

® 2013Ibnu Sina Institute. All rights reserved. http://dx.doi.org/10.11113/mjfas.v9n1.80

\section{INTRODUCTION}

Nowadays, pulp industry is the sixth largest polluter of water bodies after the oil, cement, leather, textile and steel industries[1]. The manufacture of pulp utilize large amount of fresh water which ranks third in the world, after the primary metal and the chemical industries [2]. Directly, a lot of wastewater is generated as high as $60 \mathrm{~m}^{3}$ wastewater per ton of pulp. Nearly $75 \%$ is discharged into water bodies and the rest were used for energy recovery [3].

The organic pollutants produced in Kraft and sulfite pulping processes mainly comprise compounds of lignin along with a small percentage of sucrose. It imparts brownish black colour to the water bodies and makes them unsuitable for usage in other processes. Nevertheless, when the effluents were discharged into the river,thedarkcoloration of lignin will reduces the sunlight passing through the water thus reduce the photosynthesis rate of the aquatic fluora and fauna this resulted in the reduction of the oxygen availability and adversely affects aquatic fluora and fauna[4].

Lignin is the second most abundant natural resources in the world after cellulose. Annually, approximately fifty million tons of lignins were produced as waste in the pulp industry alone. Most of the industrial lignin is being burned for the energy utilization [5]. However, the application of lignin to be burned as fuel is not economical, which can cost only up to $0.18 \mathrm{US} \$ \mathrm{~kg}$, meanwhile the uses of lignin in chemical conversion can cost as high as $1.08 \mathrm{US} \$ / \mathrm{kg}[6]$.

The new potential application of kraft lignin consists of its use as an anti-oxidant, resin, co-polymer, and a resource for carbon fibers. Besides, it also can be used in production of vanillin, animal feed pellets binder, pesticides, ect. The chemical structure of kraft lignin is shown in Fig. 1.

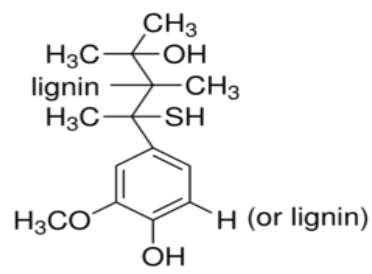

Fig. 1. Chemical structure of Kraft Lignin.

Recovery of kraft lignin from pulp industry had become more and more significant due to many application of the final product, at the same time it will solve the pollution problems. There were various methods developed for lignin removal and recovery from spent pulping liquor. These include methods such as precipitation, electrocoagulation, ultrafiltration, ion exchange, reverse osmosis, ozonation andelectrolysis [7, 8]. However, these methods have their drawbacks. For example, by using the acid precipitation, the acid sludge is corrosive and nonecofriendly. In addition, these methods are expensive and tedious especially when operated in large scale. 
Liquid membrane process has high potential in the removal of kraft lignin from simulated liquid waste solution. So far it has its main application in the field of metalextraction[9]. Besides that, it also has been certified in removing organic wastewater such as chlorophenol[10], phenylenediamine[11], benzimidazole[12] and phenol [13].It is also applied in industrial scale for recovery of phenol and zinc from wastewater.Thetreatment on mixtures of macromolecules, especially those with charged is still an attractive and challenging new field for this research. The importance of the many polyelectrolytes is the evident from both scientific and industrial points of view.

This study attempted to develop a liquid membrane formulation for kraft lignin removal from aqueous waste solution using LLE. It is the preliminary study for screening the parameters which related to ELM process.LLE is based on the principle that a solute can distribute itself in a certain ratio between immiscible solvents and extraction process depends on its mass transfer rate [14]. The extraction is based on ion-exchange reaction between a carrier dissolved in an oil phase which is kerosene and kraft lignin solution. Different affect parameters were investigated such as types of carrier, concentration of carrier, types of diluents and types of stripping agents.

\section{EXPERIMENTAL}

\subsection{Chemicals and Reagents}

All the reagents were of analytical grade and used without further purification. The following carriers were used: Trioctylamine (TOA), Tridodecylamine (TDA) from Merck (Germany), Tricaprylylmethylammonium chloride (Aliquat 336) and Tri-n-octylphosphine Oxide (TOPO) from Acros, Tributyl Phosphate(TBP) and Bis(2ethylhexyl)-Phosphat (D2EPHA) from Merck, Diisooctylthiophosphinic acid(Cyanex302) from Fluka. Solvent screening was carried out with n-dodecane from Merck (Germany) and kerosene was obtained from SigmaAldrich. Different stripping agents were used includes sodium hydroxide $(\mathrm{NaOH})$, sodium chloride $(\mathrm{NaCl})$, sodium carbonate $\left(\mathrm{Na}_{2} \mathrm{CO}_{3}\right)$, sodium bicarbonate $\left(\mathrm{NaHCO}_{3}\right)$, sodium silicate $\left(\mathrm{Na}_{2} \mathrm{SiO}_{3}\right)$, hydrochloric acid $(\mathrm{HCl})$, sulphuric acid $\left(\mathrm{H}_{2} \mathrm{SO}_{4}\right)$ which are procured from Merck (Germany). Kraft lignin (KL) was procured from SigmaAldrich.

\subsection{Liquid-liquid extraction}

The experiments on forward extraction were carried out by mixing an equal volume $(10 \mathrm{ml})$ of kraft lignin solution and organic-carrier solutions using a mechanical shaker at $320 \mathrm{rpm}$ for a period of 18 hours. The mixture was then transferred into a separating funnel with cautions. After the phase separations (around 15-30 minutes), sample of aqueous solution at the bottom was carefully separated from the organic phase. The amount of kraft lignin in the aqueous phase was determined by UV-vis spectrophotometer while that in the organic phase was obtained from the material balance. The procedures were repeated to obtain the best carrier and diluent in kraft lignin extraction. The effect of carrier concentration was investigated in order to determine the nature of kraft lignin extraction in selected extractant. The distribution ratio (D) and percentage of extraction (E) were calculated by the following formula:

$$
\begin{gathered}
D=\frac{[K L]_{\text {org }}}{[K L]_{a q}} \\
\% E=\frac{[K L]_{a q 0}-[K L]_{a q}}{[K L]_{a q 0}}
\end{gathered}
$$

where $[K L]_{\text {org }}$ is the kraft lignin concentration in the organic phase(mg/L), $[K L]_{a q 0}$ is the initial KL concentration of aqueous phase (mg/L), $[K L]_{a q}$ is the $\mathrm{KL}$ concentration of aqueous phase after extraction(mg/L).

In the stripping process, the experiment was designed to identify the best choice of stripping agent. Stripping process was the reverse extraction step. Therefore, organic phase (kraft lignin loaded carrier) was taken from extraction process and similar procedures were repeated using various stripping agents.

\subsection{Analytical Instruments}

A UV-vis spectrophotometer (Jenway 7305 Spectrophotometer) was used for measurement of kraft lignin concentration in the aqueous phase. A calibration curve has been generated using known concentration of kraft lignin within the range of $0-100 \mathrm{mgl}^{-1}$ at $280 \mathrm{~nm}$ wavelength. The FTIR spectra of the lignin samples embedded in a KBR disk were obtained with a FTIRspectrophotometer in a frequency range of 500 to $4000 \mathrm{~cm}^{-}$ 1. Fig. 2 shows the IR spectra of the kraft lignin. A strong peak at $3355 \mathrm{~cm}^{-1}$ is characteristic peak of phenolic compoundsor $\mathrm{OH}$ groups and peak at $2937 \mathrm{~cm}^{-1}$ is assigned to $\mathrm{CH}$ stretching of methyl or methylene groups. $1512 \mathrm{~cm}^{-}$ ${ }^{1}$ and $1593 \mathrm{~cm}^{-1}$ is characterized as aromatic ring while 1452 $\mathrm{cm}^{-1}$ and $1424 \mathrm{~cm}^{-1}$ have attributed to methoxyl groups. The ether-O- at $1030 \mathrm{~cm}^{-1}$ was very intense. It is more difficult to make a detailed analysis of the spectral region below $1400 \mathrm{~cm}^{-1}$. This is due to the complex bands and it contributed by various vibrational modes[15, 16].

\section{RESULTS \& DISCUSSION}

\subsection{Choice of carrier}

Selection of carrier is the most important factor in liquid-liquid extraction process. Different types of carrier were used such as acidic carrier(D2EHPA and Cyanex 302), basic carrier (TOA, TDA and Aliquat 336), and solvating carrier (TOPO and TBP). Table 1 showsthat the highest percentage of Kraft lignin (KL) extraction is 
performed by using Aliquat 336. Aliquat 336is a quaternary ammonium salt. There is no literature shows on liquidliquid extraction of KL. Kraft lignin has two kinds of ionizing groups ( $\mathrm{SH}$ and $\mathrm{OH}^{-}$). The anionic charge of Kraft lignin will react with the positive charge of Aliquat 336 in ion-exchange reaction. Thus,Aliquat 336 has been chosen as carrier in KL extraction study.

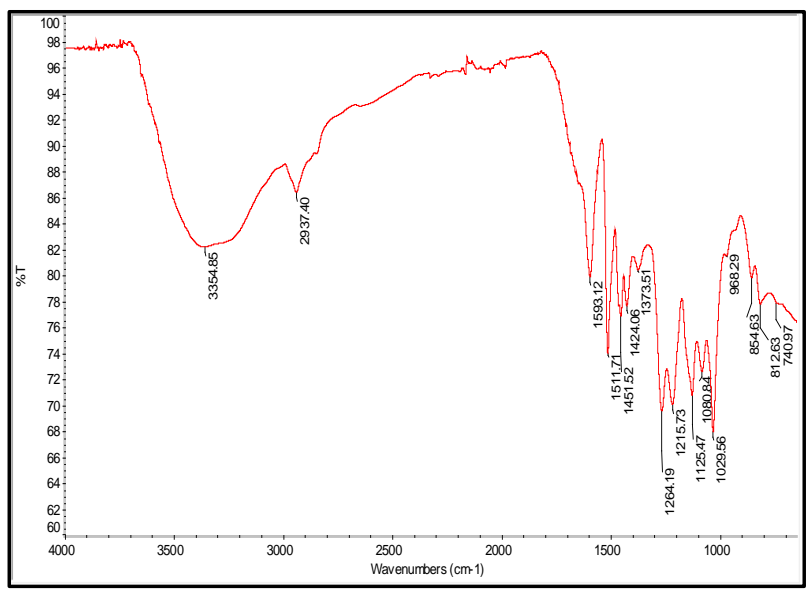

Fig. 2. FTIR spectra of kraft lignin.

\subsection{Choice of carrier}

Selection of carrier is the most important factor in liquid-liquid extraction process. Different types of carrier were used such as acidic carrier(D2EHPA and Cyanex 302), basic carrier (TOA, TDA and Aliquat 336), and solvating carrier (TOPO and TBP). Table 1 showsthat the highest percentage of Kraft lignin (KL) extraction is performed by using Aliquat 336. Aliquat 336is a quaternary ammonium salt. There is no literature shows on liquidliquid extraction of KL. Kraft lignin has two kinds of ionizing groups ( $\mathrm{SH}$ and $\mathrm{OH}^{-}$). The anionic charge of Kraft lignin will react with the positive charge of Aliquat 336 in ion-exchange reaction. Thus, Aliquat 336 has been chosen as carrier in KL extraction study.

\subsection{Choice of Diluents}

Diluents should be chosen based on the immiscibility with the aqueous solution, viscosity, specific gravity, volatility and number of carbon. The extraction was carried out usingtwo differenttypes of diluents which are kerosene and n-dodecane. The result shows in Table 2 indicate that the extraction percentage using kerosene as diluent is $93 \%$ which is higher than dodecane. This is due to the less solubility of Aliquat 336 in dodecane as observed. Additional modifier is needed if dodecane is used as diluent. Hence,kerosene is chosen as diluent due to its less toxic and low in dielectric constant (1.8) is preferred [17].
Table 1. Liquid-liquid extraction of Kraft lignin (Experimental conditions: $[\mathrm{KL}]=100 \mathrm{ppm},[$ carrier $]=0.5 \mathrm{M}$, agitation speed $=320 \mathrm{rpm}$, duration $=18 \mathrm{hrs}, \mathrm{T}=26^{\circ} \mathrm{C}$, diluents: kerosene, $\mathrm{pH}=8$, Treat ratio: 1:1).

\begin{tabular}{lll}
\hline Groups & Carrier & $\begin{array}{l}\text { \% } \\
\text { Extraction }\end{array}$ \\
\hline Basic & $\begin{array}{l}\text { Tricaprylylmethylammoniu } \\
\text { m chloride(Aliquat 336) } \\
\text { Tridodecylamine(TDA) } \\
\text { Trioctylamine(TOA) }\end{array}$ & 93 \\
\hline Solvating & $\begin{array}{l}\text { Tri-n-octylphosphine } \\
\text { Oxide(TOPO) } \\
\text { Tributyl Phosphate(TBP) }\end{array}$ & 0 \\
\hline Acidic & $\begin{array}{l}\text { Diisooctylthiophosphinic } \\
\text { acid(Cyanex 302) }\end{array}$ & 0 \\
& $\begin{array}{l}\text { Bis(2-ethylhexyl)- } \\
\text { Phosphat(D2EPHA) }\end{array}$ & 0 \\
\hline
\end{tabular}

Table 2. Effect of different diluents in liquid-liquid extraction. (Experimental conditions: $[\mathrm{KL}]=100 \mathrm{ppm}$, $[$ Aliquat 336$]=$ $0.5 \mathrm{M}$, agitation speed $=320 \mathrm{rpm}$, duration $=18 \mathrm{hrs}, \mathrm{T}=26^{\circ} \mathrm{C}$, $\mathrm{pH}=8$, Treat ratio: $1: 1)$.

\begin{tabular}{ll}
\hline Diluents & \% Extraction \\
\hline Kerosene & 93 \\
n-dodecane & 71 \\
\hline
\end{tabular}

\subsection{Effect of Carrier Concentration}

The effects of Aliquat 336 concentration within 0.003-0.3M baseon the extraction of KL from the aqueous solution were being presented in Fig. 3. The results show that the percentage of KL extraction increased drastically up to $0.1 \mathrm{M}$. Further increase the carrier concentration, the extraction percentage increase and achieves plateau. It is indicates the excess of 'free extractant'. At this region, the percentage of $\mathrm{KL}$ is almost $90 \%$. Hence, $0.1 \mathrm{M}$ of Aliquat 336 has been chosen for the next parameters.

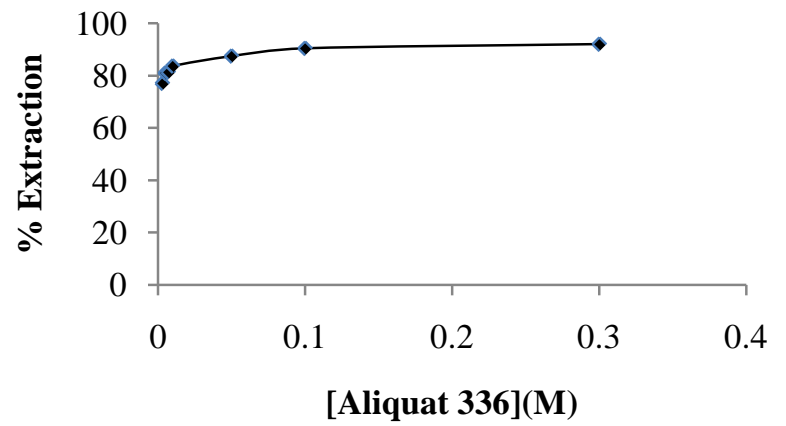

Fig. 3. Effect of carrier concentration in liquid-liquid extraction (Experiment conditions: $[\mathrm{KL}]=100 \mathrm{ppm}$, Carrier: 
Aliquat 336, agitation speed $=320 \mathrm{rpm}$, duration $=18 \mathrm{hrs}, \mathrm{T}=$ $26^{\circ} \mathrm{C}$, diluents: kerosene, $\mathrm{pH}=8$, Treat ratio: $\left.1: 1\right)$.

The nature of the extracted KL was evaluated by the plot of $\log \mathrm{D}$ versus $\log$ [Aliquat336] in Fig. 4. The slope obtained is 3.592 is nearly equal to 4 . Hence, the kraft lignin to reagent ratio was found to be 1:4. Therefore, it indicates that one mole of KL requires four mole of Aliquat 336 for extraction taken place. It justifies that bulky macromolecule of kraft lignin required large amount of carriers for extraction process.

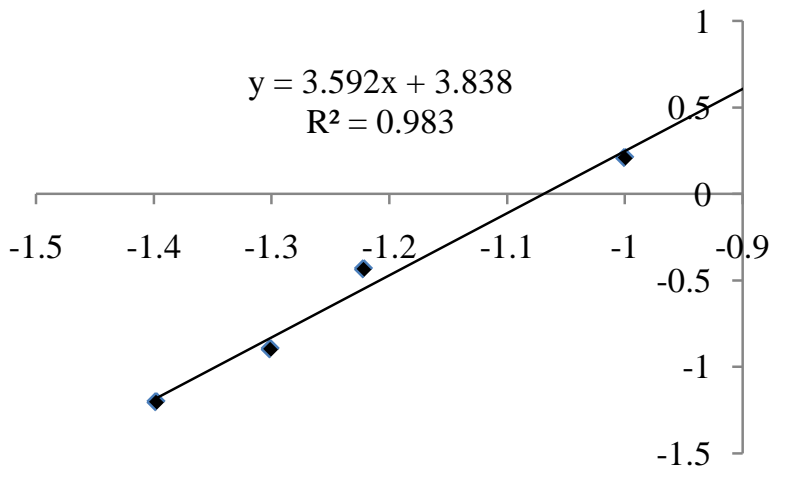

Fig. 4. Stoichiometric plot for the equilibrium extraction of $\mathrm{KL}$ using Aliquat 336 as carrier and kerosene as solvent.

\subsection{Choice of Stripping Agent}

The choice of stripping agent plays an important role in KL recovery and minimizing the co-transport of water in the extraction process. KL loaded organic solution from liquid-liquid extraction was examined in stripping process by using various types of stripping agents which are $\mathrm{NaOH}, \mathrm{NaCl}$, Sodium carbonate $\left(\mathrm{Na}_{2} \mathrm{CO}_{3}\right)$, Hydrochloric acid $(\mathrm{HCl})$, acid sulphuric $\left(\mathrm{H}_{2} \mathrm{SO}_{4}\right)$, sodium silicate $\left(\mathrm{Na}_{2} \mathrm{SiO}_{3}\right)$, sodium bicarbonate $\left(\mathrm{NaHCO}_{3}\right)$. The results illustrated in Fig. 5. From the results, the stripping percentage is not high. Higher stripping percentage goes to $\mathrm{NaOH}, \mathrm{HCl}$ and $\mathrm{NaHCO}_{3}$ which range from $15-20 \%$. Emulsion liquid membrane process that combines extraction and stripping in one step was carried on in kraft lignin removal. The validity of $\mathrm{NaOH}, \mathrm{HCl}$ and $\mathrm{NaHCO}_{3}$ as stripping agent was observed in Fig. 6. The results show that $\mathrm{NaHCO}_{3}$ achieve highest extraction which is $97 \%$ of $\mathrm{KL}$ extraction. Hence, $\mathrm{NaHCO}_{3}$ was chosen as the best stripping agent.

\section{CONCLUSION}

The method presented offers a simple approach for selective extraction of Kraft lignin for removal and recovery. It can be concluded the best liquid membrane formulation obtained is by using Aliquat 336 as carrier, kerosene as diluents and $\mathrm{NaHCO}_{3}$ as stripping agent.

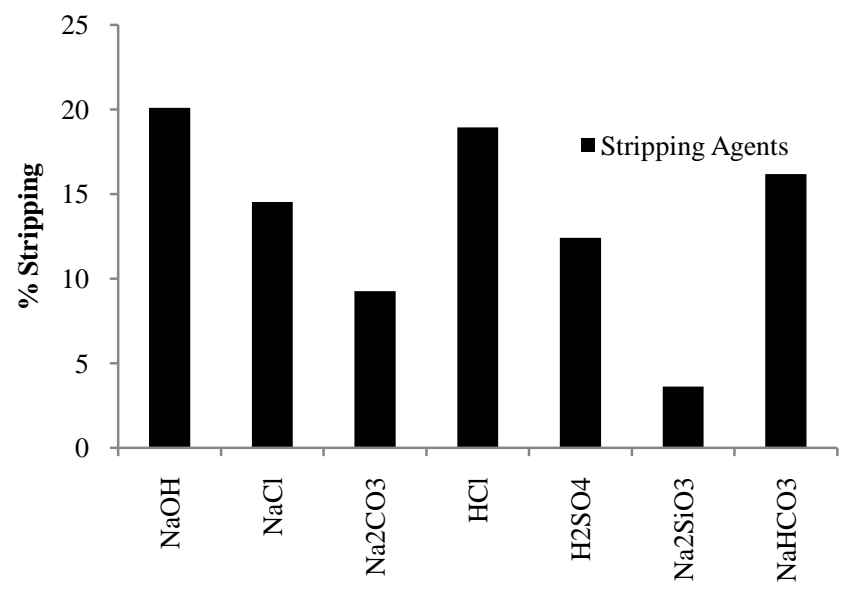

Fig. 5. Effect of different stripping agent in liquid-liquid extraction (Experiment conditions: $[\mathrm{KL}]=100 \mathrm{ppm}$, [Aliquat336] $=0.01 \mathrm{M}$, agitation speed $=320 \mathrm{rpm}$, duration = $18 \mathrm{hrs}, \mathrm{T}=26^{\circ} \mathrm{C}$, diluents: kerosene, $\mathrm{pH}=3$, Treat ratio: $1: 1$ ).

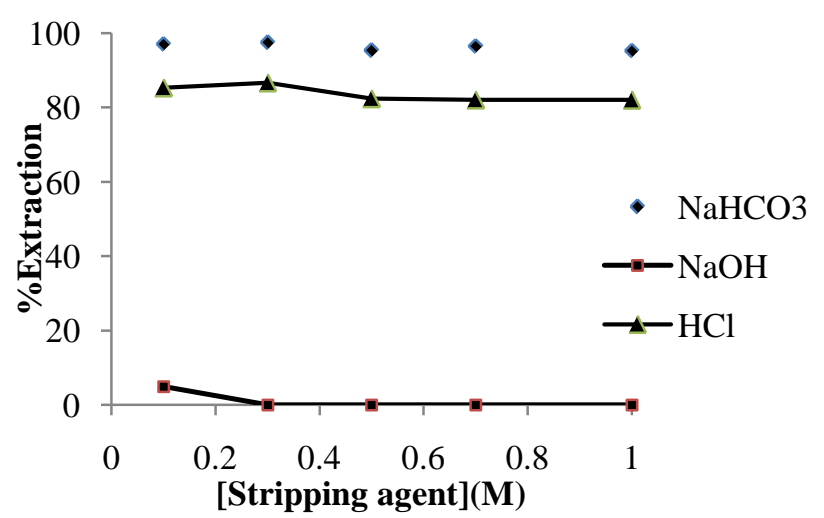

Fig. 6. Effect of types and concentration of stripping agent on kraft lignin extraction (Experimental conditions: [Aliquat 336]:0.1M, Homogenizer Speed: 12000 rpm, Emulsifying Time: 5 minutes, Initial kraft lignin Concentration: 100 ppm, Treat Ratio: 1:5, [Span 80]: 3\%w/v ,Agitation Speed: 250 rpm, Extraction Time: 5 minutes)

\section{ACKNOWLEDGEMENT}

The author would like to acknowledge Ministry of Higher Education(MOHE)and UniversitiTeknologi Malaysia (GUP:Q.J130000.7125.00H35) for financial support to make this research possible.

\section{NOMENCLATURE}

Aliquat 336 Tricaprylylmethylammonium chloride

ELM Emulsion Liquid membrane

LLE Liquid-liquid extraction

Vm volume membrane/organic phase

Vf volume feed phase

[Aliquat 336] concentration of Aliquat 336 
KL

$\mathrm{D}$

kraft lignin

distribution ratio

\section{REFERENCES}

[1] M. Ali and T.R. Sreekrishnan, Process Biochem., 36 (2000) 25-29.

[2] J. Kallas, and R. Munter, Water Sci. Technol., 29 (1994) 259-272.

[3] G. Thompson, J. Swain, M. Kay, and C.F. Forster, Bioresource Technol., 77 (2001) 275-286.

[4] C. Xiao, R. Bolton, and W.L. Pan, Bioresource Technol., 98 (2007) 1482-1488.

[5] D. Mohan, C.U. Pittman Jr, and P.H. Steele, J. Colloid Interf. Sci., 297 (2006) 489-504.

[6] A.L. Macfarlane, R. Prestidge, M.M. Farid, and J.J.J. Chen, Chem. Eng. J, 148 (2009) 15-19.

[7] G. Liu, Y. Liu, J. Ni, H. Shi, and Y. Qian, Desalination, 160 (2004) 131-141.

[8] O. Wallberg and A.-S. Jönsson, Desalination, 195 (2006) 187-200.

[9] B. Sengupta, M.S. Bhakhar, and R. Sengupta, Hydrometallurgy, 99 (2009) 25-32.
[10] C.-C. Lin and R.L. Long, Chem. Eng. Commun., 156 (1997) 45-58.

[11] L. Chuannan, X. Zhongpeng, W. Xueying, and L. Zhen, 3rd International Conference on Bioinformatics and Biomedical Engineering (ICBBE), 11-13 June 2009, Beijing, p. 1-4.

[12] S. Venkatesan and K.M. Meera Sheriffa Begum, Chem. Eng. J, 148 (2009) 254-262.

[13] Y.S. Ng, N.S. Jayakumar, and M.A. Hashim, J. Hazard. Mater., 184 (2010) 255-260.

[14] D.W. Lee, W.H. Hong, and K.Y. Hwang, Separ. Sci. Technol., 35 (2000) 1951-1962.

[15] M.N. Mohamad Ibrahim, N. Zakaria, C.S. Sipaut, O. Sulaiman, and R. Hashim, Carbohyd. Polym., 86 (2011) 112-119.

[16] N.E. El-Mansouri, Q. Yuan, and F. Huang, Bioresources Technol., 6 (2011) 2647-2662.

[17] N.E. El-Hefny, Y.A. El-Nadi, and I.M. Ahmed, Int. J. Miner. Process., 101 (2011) 58-62. 